\title{
Effect of trimethylolpropane triacrylate cross-linkages on the thermal stability and char yield of poly (methyl methacrylate) nanocomposites
}

\author{
Logan C. Hatanaka ${ }^{1}$, Qingsheng Wang², Zhengdong Cheng' and M. Sam Mannan ${ }^{1, *}$ \\ ${ }^{1}$ Mary Kay O'Connor Process Safety Center, Artie McFerrin Department of Chemical Engineering, Texas \\ A\&M University, College Station, Texas 77843-3122, USA \\ ${ }^{2}$ Department of Fire Protection \& Safety, Oklahoma State University, Stillwater, Oklahoma 74078, USA \\ *Corresponding author: M. Sam Mannan, +1 (979) 862-3985, mannan@tamu.edu
}

\begin{abstract}
Abstract

Using an in-situ polymerization method, poly (methyl methacrylate) (PMMA) cross-linked by trimethylolpropane triacrylate (TMPTA) was embedded with nanosilica, aluminum oxide, or modified montmorillonite to produce various crosslinked nanocomposites. The same three nanofillers were also embedded into PMMA without TMPTA cross-linkages to quantify the effect of TMPTA cross-linkages on the thermal stability and char yield of nanocomposites. Data from Thermogravimetric Analysis (TGA) and Derivative Thermogravimetric Analysis (DTG) were used to show that cross-linking and nanofiller content act synergistically to improve the thermal stability of PMMA, increasing the on-set of degradation by nearly $100^{\circ} \mathrm{C}$. The increase in thermal stability was attributed to the elimination of low temperature end initiated polymer unzipping by TMPTA cross-linkages and simultaneous stabilization of remaining degradation reactions due to nanofiller content. Char formed during a fire accumulates on the surface of the nanocomposite, forming a barrier that protects any unburned material below the surface. The DTG data showed nanocomposites containing $1 \mathrm{wt} \%$ silica in PMMA cross-linked by TMPTA produced $14.1 \%$ char residues, while nanocomposites without TMPTA cross-linkages required five times the mass of nanofiller to achieve similar yields.
\end{abstract}




\section{Keywords}

Nanocomposites, poly(methyl methacrylate), cross-linking, thermal stability, char yield.

\section{Introduction}

From 1977 to 2014, the total number of fire-related incidents in the United States dropped from $3,264,500$ to $1,298,000$, indicating significant progress in the ongoing effort to reduce fire losses(1). Despite this decreasing trend, the total cost due to fire damage in the US was estimated to be $\$ 329$ billion in 2011, suggesting that there is still significant room for improvement(2). The development and implementation of flameretardants for combustible and flammable materials is one step in minimizing total fire losses.

One active area in flame-retardant research is in polymeric materials. The lightweight, low cost, and high performance properties of polymers make them an ideal candidate for many industrial and household products. However, since polymers are generally composed of energy-dense hydrocarbons, their widespread application has the potential to increase the risk of fire if left unmitigated. It is important to address the flammability of polymeric materials through flame-retardants.

Many polymeric flame-retardants increase the final char yield of a burned polymer(3). During a fire, the burning polymer produces char, which accumulates as a layer on the surface of the material. This layer serves as a barrier between the unburned polymer and the fire, reducing heat transfer. Furthermore, the presence of char also indicates incomplete combustion. In a fire, the polymer first undergoes pyrolysis, breaking down into smaller, more volatile compounds that diffuse away from the 
polymer surface and burn in the vapor phase. By enhancing char yield, more mass is retained in the condensed phase where it cannot burn, reducing the total heat released in the fire. Not only can these flame-retardant polymers be used to produce inherently safer bulk plastics, they can also be applied as protective coatings, producing a sacrificial barrier in the event of a fire.

While polymeric flame-retardants have reduced the consequences associated with polymer flammability, many of these flame-retardants have received criticism over their toxicity and environmental impact. One common flame-retardant, tris (1,3dichloroisopropyl) phosphate (TDCPP) is a known neurotoxin and mutagen in animals, while other flame-retardants, such as a group of flame-retardants called pentaBDE's, are environmentally persistent pollutants(4-7). To make matters more complicated, the United States and Europe have prompted the creation of country-specific standards which require the implementation of flame-retardant materials in synthetic furniture, fabrics, and other products(8). These regulations have led to an increase in the use of flame-retardants, highlighting the need for nontoxic and non-persistent flame-retardant alternatives $(9,10)$.

In the last three decades, polymeric nanocomposites, generally consisting of inorganic nanoparticles embedded in a polymer matrix, have gained significant attention in literature as potential replacements for antiquated toxic flame-retardants. When burned, flame-retardant nanocomposites produce a physical barrier on the surface of the burning material, similar to charring flame-retardants. However, unlike traditional charring polymers, much of the char is produced by a catalytic mechanism on the surface of the nanofiller leading to the formation of an insulating physical barrier composed of carbonaceous char and agglomerated nanoparticles (11-17). In addition, unlike conventional flame-retardants, nanocomposites are generally more thermally 
stable when compared to neat polymers due to reduced polymer mobility, free-radical trapping by the nanofiller, and the replacement of combustible polymer with thermally stable nanofiller(11). Since the composition of nanocomposites can be fine tuned with different fillers and polymers, nanocomposites are a good candidate to replace antiquated toxic flame-retardants.

Previous research has been conducted utilizing polymeric nanocomposites in conjunction with traditional flame-retardants with some success. Common flameretardants such as ammonium polyphosphate (APP) were combined with nanoparticles in a polymeric matrix to produce synergistic flame-retardant systems with enhanced char yields and increased thermal stability(18-20). Similar to APP and other flameretardants, cross-linking has been previously shown to increase the char-yield of polymers, but the thermal stability effects of common cross-linking agents in conjunction with nanofillers have not been adequately studied(21).

In this work, the thermal stability and char yield of cross-linked polymer nanocomposites is studied using poly (methyl methacrylate) (PMMA) cross-linked with trimethylopropane triacrylate (TMPTA). Three different nanofillers, montmorillonite (MMT), aluminum oxide ( $\mathrm{AO})$, and nanosilica, are used to observe the effect nanofiller composition has on thermal stability and char yield of TMPTA cross-linked PMMA. In addition, linear (not cross-linked) nanocomposites were produced to compare with the cross-linked materials. 


\section{Methods}

\section{Materials}

The monomer, methyl methacylate (MMA), and the initiator, 1,1'azobis(cyclohexanecarbonitrile) (ABCN), is supplied by Polysciences. The nanofillers, which include montmorillonite nanoclay sheets organically modified with aminopropyltriethoxysilane (MMT, nanoscale thickness, $<20$ micron width), spherical aluminum oxide nanoparticles ( $\mathrm{AO}, 13 \mathrm{~nm}$ primary particle size), and spherical nanosilica (10-20nm primary particle size), are purchased from Sigma-Aldrich. The cross-linking agent, trimethylolpropane triacrylate (TMPTA) is also supplied by SigmaAldrich. X-Ray Diffraction was conducted using a Bruker D8 power diffractometer and $\mathrm{Cu}$ k- $\alpha$ radiation source to initially observe the dispersion of nanocomposite samples. However, tested samples appeared similar to their polymeric counterparts.

\section{Nanocomposite synthesis}

Nanocomposites are produced using an in-situ method. One of the three nanofillers (MMT, AO, or silica) is massed and added to the MMA monomer in a sealed glass vessel so that concentration of nanofiller in the final nanocomposite is $1 \mathrm{wt} \%$, $3 \mathrm{wt} \%$, or $5 \mathrm{wt} \%$. For cross-linked samples, the cross-linking agent, TMPTA, is added to the solution in a 1:60 molar ratio of TMPTA:MMA. No TMPTA is added for linear samples. This solution is mixed using a magnetic stirring bar for 30 minutes, followed by ultrasonication at $20^{\circ} \mathrm{C}$ for an additional 30 minutes. After ultrasonication, $\mathrm{ABCN}$ initiator is massed and added to the solution so that the initiator concentration is equal to $0.5 \%$ of the total monomer and cross-linker mass $(0.5 \%$ of the mass of MMA and TMPTA combined). A magnetic stirring bar is also added to the vessel before it is sealed tightly using a lid with a silicone septum. Two needles are punctured through the silicone septum to begin inerting. One needle bubbles nitrogen through the solution, 
while the second prevents overpressurization of the glass vessel. The vessel is then transferred to an oil bath which maintains the polymerization temperature at $70^{\circ} \mathrm{C}$. A hot plate with a magnetic stirrer is used to continually stir both the glass reaction vessel and the oil bath simultaneously. Polymerization proceeds until approximately five minutes before the solution gels, at which point the partially polymerized solution is transferred to small polypropylene vials. Polyethylene insulation foam is used to keep the small polypropylene vials buoyant as they float in the oil bath for an addition 48 hours to complete curing, after which the materials are removed from their molds and tested.

\section{Nanocomposite characterization}

Thermogravimetric Analysis (TGA) and Derivative Thermogravimetric (DTG) studies was conducted using a Mettler Toledo TGA/DSC 1. TGA was used to measure mass loss with respect to temperature while DTG was used to measure mass loss rate with respect to temperature. This was done under a nitrogen atmosphere and at a ramp rate of $10^{\circ} \mathrm{C} / \mathrm{min}$. Samples were roughly $5 \mathrm{mg}$ in mass and roughly spherical in shape and repeated tests show reproducible results. These data are used to measure the onset of degradation, the char yield, and the general degradation behavior of the nanocomposites produced.

\section{Results and discussions}

Nanocomposites cross-linked by TMPTA with Silica, MMT, and AO nanofillers were tested using TGA and compared with the results from linear nanocomposites to quantify how PMMA cross-linking and nanofiller content interact to affect thermal stability and char yields. In general the low loading (1wt $\%$ nanofiller) samples are homogenous, with increasing levels of agglomeration visually apparent at higher 
loadings. The results for each nanofiller will be discussed separately and then compared to understand how differences in nanoparticle structure and chemistry may affect degradation.

\section{Thermal stability and char yield of silica nanocomposites}

Figure 1(A) presents the TGA data for linear $1 \mathrm{wt} \%$ silica nanocomposites and $1 \mathrm{wt} \%$ silica nanocomposites cross-linked by TMPTA, while Figure 2 shows the corresponding DTG curves. The TGA data for linear PMMA without nanofiller and PMMA cross-linked by TMPTA without nanofiller are also included in Figure 1(A). The degradation of linear PMMA and 1wt\% silica in linear PMMA occurs in a two-step process, indicated in Figure 1(A) by two sudden drops in the mass as temperature increases or equivalently in Figure 2 as two mass loss peaks. The first mass loss begins near $200^{\circ} \mathrm{C}$, and corresponds to unsaturated-end initiated polymer unzipping, while the second mass loss begins near $300^{\circ} \mathrm{C}$, and corresponds to random depolymerization, shown in previous studies(22).

In contrast, cross-linked PMMA and 1wt $\%$ silica nanocomposites cross-linked by TMPTA only have one major degradation region, shown in Figure 1(A) as a single drop in mass as temperature increases and in Figure 2 as a single mass loss peak. The DTG for linear and cross-linked 1wt $\%$ silica nanocomposites in Figure 2 clearly shows the low temperature mass loss peak centered at $250^{\circ} \mathrm{C}$ is absent in the cross-linked nanocomposite. This indicates that the presence of TMPTA cross-linkages stabilize unsaturated-end initiated polymer unzipping, shown in previous studies(21). In addition, the remaining high temperature mass loss peak centered near $370^{\circ} \mathrm{C}$ in Figure 2 is shifted upwards in the cross-linked nanocomposite, indicating nanosilica shifts degradations to higher temperatures. This is typical of either a reduction in the mobility 
of individual polymer chains by nanofiller, stabilizing the polymer chain or a radical trapping mechanism(11).

\section{[INSERT FIGURE 1 HERE WITH CAPTION]}

\section{[INSERT FIGURE 2 HERE WITH CAPTION]}

Similar behavior is seen from the TGA measurements with $3 \mathrm{wt} \%$ and $5 \mathrm{wt} \%$ silica nanocomposites, shown in Figure 1(B) and 1(C), respectively. All linear nanocomposites degrade in two mass loss steps, while cross-linked nanocomposites degrade in a single step. In addition, the single mass loss step in cross-linked nanocomposites occurs at elevated temperature when compared to cross-linked PMMA.

The onset of degradation, defined here as the temperature at $5 \%$ mass loss, is dramatically improved in all silica nanocomposites. However, silica nanocomposites cross-linked by TMPTA outperformed linear silica nanocomposites without crosslinking drastically. As shown in Figure 1(A), cross-linked PMMA showed a $20^{\circ} \mathrm{C}$ increase in the onset of degradation compared to linear PMMA, while a linear 1wt $\%$ silica nanocomposite showed a $9^{\circ} \mathrm{C}$ increase in the onset of degradation. For the crosslinked $1 \mathrm{wt} \%$ silica nanocomposites, the onset of degradation was increased dramatically by $91^{\circ} \mathrm{C}$, significantly more than either silica or cross-linked PMMA separately. The cross-linked $3 \mathrm{wt} \%$ and $5 \mathrm{wt} \%$ silica nanocomposites in Figure 1(B) and 1(C) show a similar dramatic effect, with a maximum enhancement to the onset of degradation by $96^{\circ} \mathrm{C}$.

The increased onset of degradation is explained by two simultaneous effects, which have previously been mentioned in this section. Firstly, polymer cross-linking 
stabilizes low temperature reactions, leading to one-step degradation. Secondly, the addition of silica delays degradation reactions to higher temperatures because of reduced polymer mobility or free radical trapping. These simultaneous effects provide drastically higher onset degradation temperatures than either silica nanocomposites or cross-linked polymers separately.

The final residuals from TGA at high temperatures, labeled as char, are drastically increased in $1 \mathrm{wt} \%$ silica nanocomposites cross-linked by TMPTA. Figure 1(A) shows that the linear PMMA sample produced only a small amount of char, with only $0.8 \%$ of the original mass remaining after TGA tests. Cross-linked PMMA had a slight enhancement with $2.8 \%$ char yield, while the linear $1 \mathrm{wt} \%$ silica nanocomposite showed even greater enhancement with $6.8 \%$ char yield. However, the use of silica with cross-linked PMMA showed drastic synergistic improvements, producing a char yield of $14.1 \%$ for a $1 \mathrm{wt} \%$ silica nanocomposite cross-linked by TMPTA. This is significantly greater than the char yield of cross-linked PMMA or silica nanocomposites separately. The synergistic interaction on char yield can be explained by reduced internal mass transfer in cross-linked polymers, reported previously(21). As internal mass transfer is reduced, volatile degradation products take longer to diffuse out of the material and into the vapor-space. This allows reactants to spend more time near the surface of nanoparticles, where char-forming reactions occur(23).

For TGA measurements of 3wt $\%$ silica nanocomposites in Figure 1(B), char yields of linear nanocomposites and TMPTA cross-linked nanocomposites are similar, with $11.8 \%$ and $11.2 \%$, respectively. In this case, cross-linking has a neutral effect on silica nanocomposite char yields. At a higher loading of $5 \mathrm{wt} \%$ silica in PMMA, shown in Figure 1(C), the char yields of linear nanocomposites and cross-linked 
nanocomposites changes to $14.4 \%$ and $10.0 \%$, respectively, indicating cross-linking has an antagonistic effect on the $5 \mathrm{wt} \%$ silica nanocomposites tested.

Synergistic enhancements to char yield are lost once silica loading is increased past $1 \mathrm{wt} \%$. This is explained through increased agglomeration and phase separation in cross-linked nanocomposites. During in-situ polymerization, TMPTA cross-linked materials gel at much lower degree of conversion, reducing the effective amount of mixing during polymerization and increasing the amount of nanoparticle phase separation. With more agglomeration in cross-linked nanocomposites, a smaller fraction of nanoparticle surface is exposed to the polymer, reducing the amount of char forming reactions that occur at the polymer-nanoparticle interface.

\section{Thermal stability and char yield of MMT nanocomposites}

Nancomposites cross-linked by TMPTA were also produced using organically modified MMT as a nanofiller and compared with linear MMT nanocomposites. The TGA results for nanocomposites with $1 \mathrm{wt} \%, 3 \mathrm{wt} \%$, and $5 \mathrm{wt} \% \mathrm{MMT}$, are shown in Figure 3(A), 3(B) and 3(C), respectively. Similar to the silica nanocomposites in this study, all linear MMT nanocomposites degrade in a two-step fashion, while all TMPTA cross-linked MMT nanocomposites degrade in a single step.

\section{[INSERT FIGURE 3 WITH CAPTION]}

The temperature at which the onset of degradation occurs in MMT nanocomposites cross-linked by TMPTA is elevated dramatically, with a maximum increase of $97^{\circ} \mathrm{C}$ compared to linear PMMA. This is similar to the cross-linked silica nanocomposites previously discussed, and is likely explained by the same two 
simultaneous complimentary factors. The single-step degradation caused by crosslinking nearly eliminates low temperature unsaturated-end initiated polymer unzipping, while nanofiller content reduces polymer mobility and traps free radicals, simultaneously increasing the temperature at which the remaining degradation reactions occur.

The char yield of MMT nanocomposites is dependent on TMPTA cross-linkages and MMT loading. For 1wt $\%$ MMT nanocomposites in Figure 3(A), the char yields for linear and cross-linked materials are similar (5.2\% and 5.8\%, respectively). This indicates TMPTA cross-linking has a neutral effect on char yield for the $1 \mathrm{wt} \%$ MMT nanocomposites tested. For 3wt\% MMT nanocomposites in Figure 3(B), the char yields of linear nanocomposites and cross-linked nanocomposites are again similar with 7.0\% and $6.0 \%$, respectively, indicating a neutral effect. However, for $5 \mathrm{wt} \% \mathrm{MMT}$ nanocomposites in Figure 3(C), char yields of cross-linked materials are significantly improved, showing $15.2 \%$ yield compared to $11.1 \%$ with a linear $5 \mathrm{wt} \%$ MMT nancomposite, indicating a synergistic improvement to char yield.

In contrast to silica nanocomposites previously discussed, increasing the particle loading of TMPTA cross-linked MMT nanocomposites enhanced char yield. This is again explained through a reduction of the internal mass transfer and particle agglomeration. At low loadings, bulky MMT sheets reduce the internal mass transfer of PMMA significantly. Therefore, the introduction of cross-linkages is redundant to the reduction in mass transfer and provides a neutral effect. However, as MMT loading increases, MMT agglomeration and phase separation effectively reduce the number of individual agglomerates present in the material, making MMT less effective at reducing the internal mass transfer of PMMA. In this case, the introduction of cross-linkages 
further reduces the internal mass transfer in PMMA, resulting in a synergistic improvement to char yields.

\section{Thermal stability and char yield of AO nanocomposites}

In addition to silica and MMT, AO was embedded in linear PMMA and PMMA cross-linked by TMPTA. The TGA results for $1 \mathrm{wt} \%$, $3 \mathrm{wt} \%$, and $5 \mathrm{wt} \%$ AO nanocomposites are shown in Figure 4(A), 4(B), and 4(C), respectively. Linear AO nanocomposite degrade in two steps, while cross-linked AO nanocomposite degrade in a single step, similar to both MMT and silica nanocomposites in this work.

\section{[INSERT FIGURE 4 HERE WITH CAPTION]}

In addition, cross-linked $\mathrm{AO}$ nanocomposites show a significant increase to the temperature of degradation onset, with a maximum increase of $99^{\circ} \mathrm{C}$ for a cross-linked $3 \mathrm{wt} \% \mathrm{AO}$ nanocomposite. The increase in the onset of degradation is attributed to the same two simultaneous effects as with silica and MMT nanocomposites. Cross-linking eliminates low-temperature degradations, while nanofiller content stabilizes the remaining degradation step, moving it to even higher temperatures.

The char yield of linear and TMPTA cross-linked $1 \mathrm{wt} \%$ AO nancomposites in Figure $4(\mathrm{~A})$ are similar (5.7\% and $6.1 \%$, respectively), indicating that polymer crosslinking has a neutral effect on char yields in the tested $1 \mathrm{wt} \% \mathrm{AO}$ nanocomposites. The cross-linked nanocomposites in Figure 4(B) and 4(C) show less char yield than linear nanocomposites of the same loading, indicating that cross-linking has an antagonistic effect on char yields for the $3 \mathrm{wt} \%$ and $5 \mathrm{wt} \% \mathrm{AO}$ nanocomposites tested. For $\mathrm{AO}$ nanocomposites cross-linked by TMPTA, particle agglomeration is visually more 
pronounced than in linear $\mathrm{AO}$ nanocomposites without cross-linking, causing a significant reduction in nanofiller surface area, and thus char yield.

\section{Summary of thermal stability and char yield studies}

Nanocomposites cross-linked by TMPTA have significant improvements to thermal stability when compared to linear PMMA, PMMA cross-linked by TMPTA, and linear PMMA nanocomposites without cross-linking, as summarized in Table 1. Firstly, the temperature at degradation onset is drastically increased for all cross-linked nanocomposites observed, with a maximum improvement of $99^{\circ} \mathrm{C}$. When compared to cross-linked PMMA and linear nanocomposites, every cross-linked nanocomposite tested had dramatically higher onset of degradation, indicating TMPTA cross-linking has a synergistic effect on the thermal stability of PMMA nanocomposites.

\section{[INSERT TABLE 1 HERE]}

As previously discussed, the increase in the onset of degradation is due to the two simultaneous effects. Firstly, TMPTA cross-linkages eliminate the low temperature end initiated unzipping reaction, leaving only one mass loss step. Secondly, the presence of nanofiller reduces polymer mobility and traps free radicals, stabilizing the single remaining degradation step and shifting it towards higher temperatures. The resulting effect appears to be independent of the nanofiller used.

In addition, the behavior of char yield in TMPTA cross-linked nanocomposites is complex. In some cross-linked samples, such as $1 \mathrm{wt} \%$ silica in cross-linked PMMA, the char yield was significantly higher than with linear $1 \mathrm{wt} \%$ silica nanocomposites without cross-linking, indicating TMPTA cross-linking had a synergistic effect on the char yield 
of the nanocomposites. In other cases, cross-linked nanocomposites produced char yields similar to linear nanocomposites, such as 1wt\% MMT in cross-linked PMMA. In these cases TMPTA cross-linkages had a neutral effect on char yield in nanocomposites. Finally, some cross-linked nanocomposites had a lower char yield when compared to linear nanocomposites, such as with 5wt\% AO in cross-linked PMMA. In these cases, TMPTA cross-linking had an antagonistic effect on char yield in nanocomposites. The synergistic, neutral, and antagonistic effects of polymer cross-linking in PMMA nanocomposites are summarized in Table 2.

The complex char yield behavior is attributed to both agglomeration of nanoparticles and a reduction of internal mass transfer in the nanocomposite. Crosslinking PMMA with TMPTA causes a reduction in internal mass transfer by introducing bulky cross-linkages(21). The reduction of internal mass transfer effectively increases the amount of time degradation products spend near nanoparticle surfaces where charforming reactions occur, increasing the char yield. However, since TMPTA cross-linked nanocomposites gel faster than linear nanocomposites, there is less time for mixing, resulting in greater amounts of agglomeration and phase separation in TMPTA crosslinked nanocomposites. Therefore, obtaining high char yields in cross-linked nanocomposites relies on a balance between reduced internal mass transfer and increased nanoparticle agglomeration. Medium and large scale tests, such as those in Cone Calorimetry, could give further insight into these phenomena. 


\section{Conclusions}

Linear poly (methyl methacrylate) (PMMA) nanocomposites and PMMA nanocomposites cross-linked with TMPTA were synthesized using an in-situ bulk polymerization technique and characterized using Thermogravimetric Analysis (TGA) and Derivative Thermogravimetric Analysis (DTG). Both linear and cross-linked PMMA were embedded with silica, organically modified montmorillonite (MMT), and aluminum oxide $(\mathrm{AO})$ nanoparticles separately to quantify the interaction between polymer cross-linking and nanoparticle content. TGA results showed that TMPTA cross-linked nanocomposites have drastic improvements to thermal stability when compared to linear nanocomposites. These effects showed that cross-linking and nanoparticle content work together to synergistically increase on-set of degradation by nearly $100^{\circ} \mathrm{C}$. In addition, nanocomposites cross-linked by TMPTA were shown to dramatically increase char yield when compared to linear nanocomposites without cross-linkages. Cross-linked PMMA with $1 \mathrm{wt} \%$ silica produced $14.1 \%$ char, similar to linear PMMA with 5wt\% MMT, silica, or AO. In this case, utilizing the common crosslinking agent TMPTA in conjunction with nanofiller increases the char yield of PMMA drastically, while only using one-fifth of the expensive nanoparticle component.

\section{Acknowledgements}

This work was supported by the National Science Foundation Grant Number CBET1336162.

\section{References}

1. Haynes HJG. Fire Loss in the United States. National Fire Protection Association, 2015.

2. Hall JR. Total Cost of Fire. National Fire Protection Association, 2014. 
3. Gilman JW, Kashiwagi T, Harris RH, Lomakin S, Lichtenhan JD, Bolf A, Jones P. Chemistry and Technology of Polymer Additives. Malden, MA: Blackwell Science Inc.; 1999.

4. Blum A, Ames BN. Flame-retardant additives as possible cancer hazards. Science. 1977;195(4273):17-23.

5. Dishaw LV, Powers CM, Ryde IT, Roberts SC, Seidler FJ, Slotkin TA, Stapleton HM. Is the PentaBDE replacement, tris (1,3-dichloropropyl) phosphate (TDCPP), a developmental neuro- toxicant? Studies in PC12 cells. . Toxicol Appl Pharmacol 2011;256(3):281-9.

6. Kelly BC, Ikonomou MG, Blair JD, Morin AE, Gobas FAPC. Food Web-Specific Biomagnification of Persistent Organic Pollutants. Science. 2007;317(5835):236-9.

7. Gold MD, Blum A, Ames BN. Another flame-retardant, tris- (1,3-dichloro-2-propyl)phosphate, and its expected metabolites are mutagens. . Science. 1978;200(4343):7857.

8. EFRA. Flame Retardants: Commonly Asked Questions. Brussels2007.

9. Stapleton HM, Sharma S, Getzinger G, Ferguson PL, Gabriel M, Webster TF, Blum A. Novel and High Volume Use Flame Retardants in US Couches Reflective of the 2005 PentaBDE Phase Out. . Environmental Science \& Technology. 2012;46(24):13432-9.

10. Stapleton HM, Klosterhaus S, Keller A, Ferguson PL, Bergen Sv, Cooper E, Webster TF, Blum A. Identification of Flame Retardants in Polyurethane Foam Collected from Baby Products. Environmental Science \& Technology. 2011;45(12):5323-31.

11. Chrissafis K, Bikiaris D. Can nanoparticles really enhance thermal stability of polymers? Part I: An overview on thermal decomposition of addition polymers. Thermochimica Acta. 2011;523:1-24.

12. Jash P, Wilkie CA. Effects of surfactants on the thermal and fire properties of poly(methyl methacrylate)/clay nanocomposites. Polymer Degradation and Stability. 2005;88:401-6.

13. Kandare E, Deng H, Wang D, Hossenlopp JM. Thermal stability and degradation kinetics of poly(methyl methacrylate)/layered copper hydroxy methacrylate composites. Polymers For Advanced Technologies. 2006:312-9.

14. Manzi-Nshuti C, Hossenlopp JM, Wilkie CA. Comparative study on the flammability of polyethylene modified with commercial fire retardants and a zinc aluminum oleate layered double hydroxide. Polymer Degradation and Stability. 2009;94:782-8.

15. Vyazovkin S, Dranca ID, Fan X, Abvincula R. Kinetics of the Thermal and ThermoOxidative Degradation of a Polystyrene-Clay Nanocomposite. Macromolecules Rapid Communication. 2004:498-503.

16. Gilman JW. Flammability and thermal stability studies of polymer layered-silicate (clay) nanocomposites. Applied Clay Science. 1999;15:31-49. 
17. Kashiwagi T, R.H. Harris J, Zhang X, Briber RM, Cipriano BH, Raghavan SR, Awad WH, Shields JR. Flame retardant mechanism of polyamide 6-clay nanocomposites. Polymer. 2004;45(3):881-91.

18. Cinausero N, Azema N, Lopez-Cuesta JM, Cochez M, Ferriol M. Synergistic effect between hydrophobic oxide nanoparticles and ammonium polyphosphate on fire properties of poly(methyl methacrylate) and polystyrene. . Polymer Degradation and Stability. 2011;96(8):1445-54.

19. Laachachi A, Cochez M, Leroy E, Ferriol M, Lopez-Cuesta JM. Fire retardant systems in poly(methyl methacrylate): Interactions between metal oxide nanoparticles and phosphinates. Polymer Degradation and Stability 2007;92(1):61-9.

20. Friedrich B, Laachachi A, Ferriol M, Ruch D, Cochez M, Toniazzo V. Improvement of Thermal Stability and Fire Behaviour of pmma by a (Metal Oxide Nanoparticles/Ammonium Polyphosphate/ Melamine Polyphosphate) Ternary System. Integrated Systems, Design and Technology 2010. 2011:47-58.

21. Lomakin SM, Brown JE, Breese RS, Nyden MR. An investigation of the thermal stability and char-forming tendency of cross-linked poly(methyl methacrylate). Polymer Degradation and Stability. 1993;41:229-43.

22. Kashiwagi T, Inaba A, Brown JE, Hatada K, Kitayama T, Masuda E. Effects of Weak Linkages onthe Thermal Oxidative Degradation of Poly(methyl methacrylates). Macromolecules. 1986;19:2160-8.

23. Ray SS. Clay Containing Polymer Nanocomposites: From Fundamentals to Real Applications. Oxford: Elsevier; 2013. 


\section{Figure Legends}

Figure 1(A, left): TGA results for $1 \mathrm{wt} \%$ silica nanocomposites, (B, center): $3 \mathrm{wt} \%$ silica nanocomposites, (C, right): 5wt\% silica nanocomposites.

Figure 2: DTG results for $1 \mathrm{wt} \%$ silica nanocomposites.

Figure 3(A, left): TGA results for linear 1wt $\%$ MMT nanocomposites, (B, center): $3 w t \%$ MMT nanocomposites, (C, right): 5wt\% MMT nanocomposites.

Figure 4(A, left): TGA results for linear $1 \mathrm{wt} \% \mathrm{AO}$ nanocomposites, (B, center): $3 \mathrm{wt} \% \mathrm{AO}$ nanocomposites, (C, right): $5 \mathrm{wt} \% \mathrm{AO}$ nanocomposites. 


\section{Text for Table of Content Graphic}

By utilizing cross-linking agents in conjunction with embedded nanoparticles, it is possible to produce thermally stable plastics which are capable of producing large amounts of partially combusted char, excellent for flame-retardant applications. 

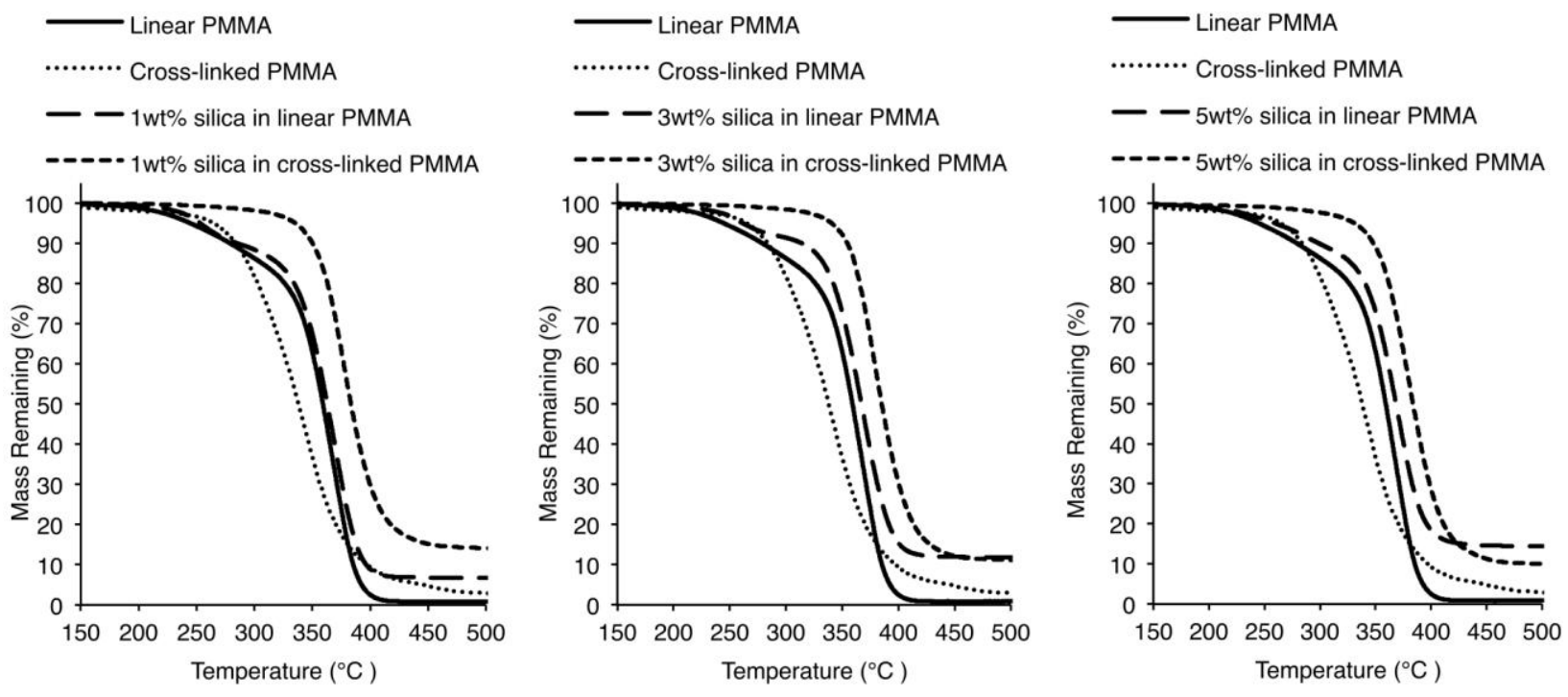


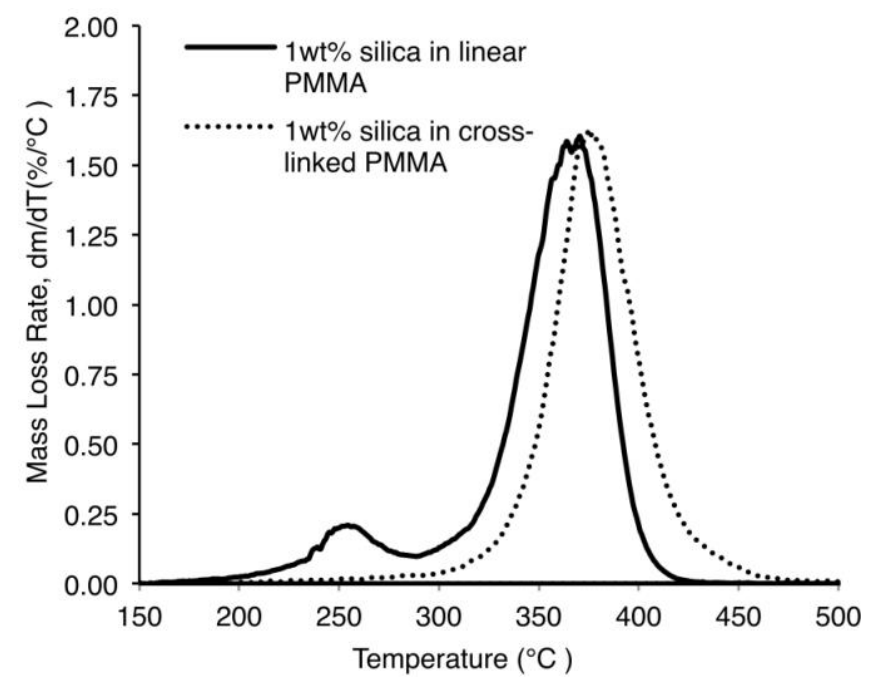



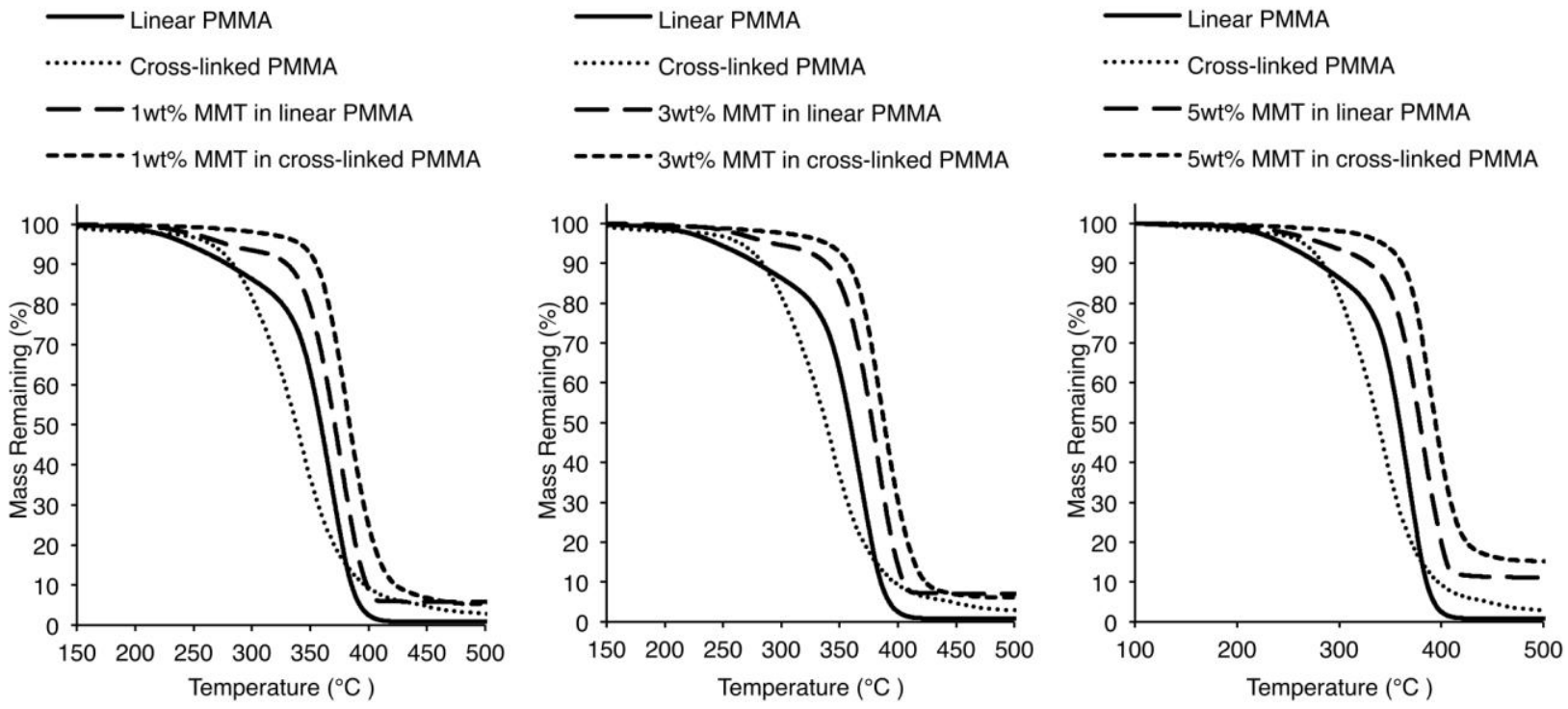

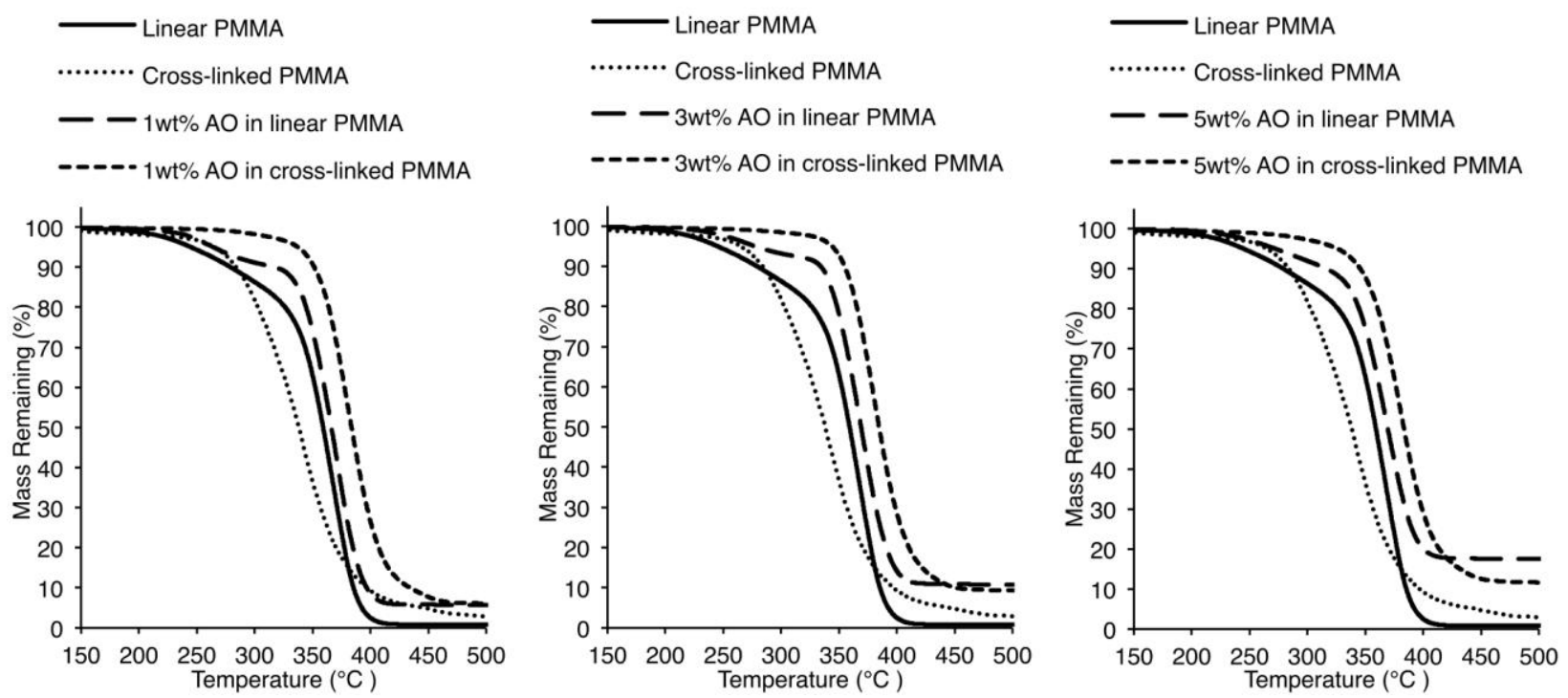
Table 1: Thermal Stability and Char Yield

\begin{tabular}{|c|c|c|c|c|}
\hline Particle & $\begin{array}{l}\text { Linear/ Cross- } \\
\text { Linked }\end{array}$ & $\begin{array}{c}\text { Particle } \\
\text { Loading (wt } \%)\end{array}$ & $\begin{array}{l}\text { Temperature at Onset of } \\
\text { Degradation }\left({ }^{\circ} \mathrm{C}\right)\end{array}$ & Char Yield (\%) \\
\hline none & linear & 0 & 245 & 0.8 \\
\hline none & cross-linked & 0 & 265 & 2.9 \\
\hline silica & linear & 1 & 253 & 6.7 \\
\hline silica & linear & 3 & 262 & 11.8 \\
\hline silica & linear & 5 & 259 & 14.4 \\
\hline silica & cross-linked & 1 & 335 & 14.1 \\
\hline silica & cross-linked & 3 & 341 & 11.2 \\
\hline silica & cross-linked & 5 & 331 & 10.0 \\
\hline MMT & linear & 1 & 278 & 5.8 \\
\hline MMT & linear & 3 & 293 & 7.0 \\
\hline MMT & linear & 5 & 284 & 11.1 \\
\hline MMT & cross-linked & 1 & 342 & 5.2 \\
\hline MMT & cross-linked & 3 & 339 & 6.1 \\
\hline MMT & cross-linked & 5 & 342 & 15.2 \\
\hline $\mathrm{AO}$ & linear & 1 & 264 & 5.7 \\
\hline $\mathrm{AO}$ & linear & 3 & 277 & 10.8 \\
\hline $\mathrm{AO}$ & linear & 5 & 272 & 17.5 \\
\hline $\mathrm{AO}$ & cross-linked & 1 & 337 & 6.1 \\
\hline $\mathrm{AO}$ & cross-linked & 3 & 344 & 9.3 \\
\hline $\mathrm{AO}$ & cross-linked & 5 & 326 & 11.6 \\
\hline
\end{tabular}

Table 2: Effect of TMPTA Cross-Linkages on Nanocomposite Char Yield: Synergistic, Neutral, and Antagonistic Effects

\begin{tabular}{cccc}
\hline Nanoparticle & $1 w t \%$ Filler & $3 w t \%$ Filler & $5 w t \%$ Filler \\
\hline Silica & Synergistic & Neutral & Antagonistic \\
MMT & Neutral & Neutral & Synergistic \\
AO & Neutral & Antagonistic & Antagonistic
\end{tabular}




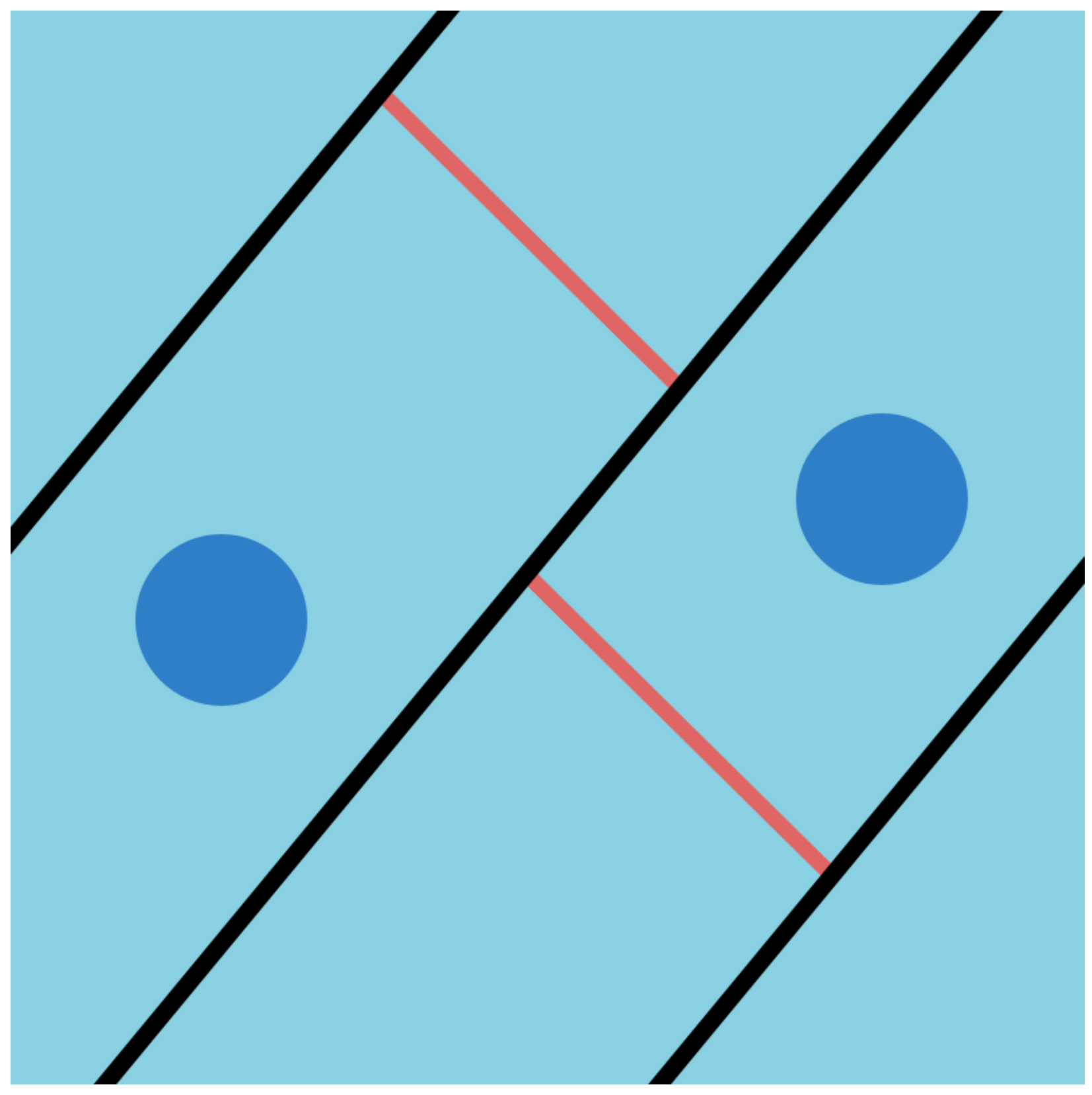

